increased (increase of FVC of more than $5 \%$ ). The skin score was reliably decreased from $11,2 \pm 9,3$ to $6,2 \pm 4,9 \quad(p<0.0001)$, FVC was increased from $76,9 \pm 19,9$ to $84,7 \pm 20,9 \% \quad(p<0.0001)$, also the increase of DLCO from $46,3 \pm 18,3$ to $47,8 \pm 16,9 \%$ from the proper amount was detected. The Valentini Disease Activity index reliably reduced from $2,93 \pm 1,7$ to $1,38 \pm 1,2$ points $(p<0,0001)$. Under the RTX therapy, the decrease of glucocorticoids dose was insignificant, but statistically reliable. Thus, prednisolone dose reduction from $11,8 \pm 4,4$ to $9,22 \pm 3,2 \mathrm{mg} /$ day was achieved Clinically insignificant but reliable decline of eGFR from $100,2 \pm 23$ to 94,6 $\pm 22 \mathrm{ml} / \mathrm{min} / 1,73 \mathrm{~m} 2$ was detected.

Conclusion: Thus, RTX has a positive influence on the basic parameters of SSc, mainly on skin damage and lung functionality, reduces the general disease activity. The influence of the RTX on kidney function requires further long-term study.

Disclosure of Interests: None declared

DOI: 10.1136/annrheumdis-2019-eular.6700

\section{AB0652 QTC INTERVAL PROLONGATION IN A SCANDINAVIAN COHORT OF PATIENTS WITH IDIOPATHIC INFLAMMATORY MYOPATHIES AND SYSTEMIC SCLEROSIS: CORRELATIONS WITH CLINICAL VARIABLES}

Sine Søndergaard Korsholm ${ }^{1}$, Maryam Dastmalchi ${ }^{2}$, John Bonde Knudsen ${ }^{1}$, Axel Cosmus Diederichsen ${ }^{3}$, Ingrid E. Lundberg ${ }^{4}$, Daniel C. Andersson ${ }^{5}$, Nanna Witting ${ }^{6}$, Søren Jacobsen ${ }^{7}$, Tina Friis ${ }^{8}$, Markus E. Krogager ${ }^{6}$, Louise Pyndt Diederichsen ${ }^{1,7}$. ${ }^{1}$ Odense University Hospital, Department of Rheumatology, Odense, Denmark; ${ }^{2}$ Karolinska University Hospital, Department of Medicine, Rheumatology Unit, Stockholm, Sweden; ${ }^{3}$ Odense University Hospital, Department of Cardiology, Odense, Denmark; ${ }^{4}$ Karolinska Institutet, Department of Medicine, Rheumatology Unit, Stockholm, Sweden; ${ }^{5}$ Karolinska Institutet, Department of Physiology and Pharmacology, Stockholm, Sweden; ${ }^{6}$ Rigshospitalet, Department of Neurology, Copenhagen, Denmark; ${ }^{7}$ Rigshospitalet, Copenhagen University Hospital, Center for Rheumatology and Spine Diseases, Copenhagen, Denmark ${ }^{8}$ Statens Serum Institut, Department of Autoimmunology and Biomarkers, Copenhagen, Denmark

Background: Idiopathic inflammatory myopathies (IIM) are rare devastating diseases characterized by progressive muscle weakness and muscle fatigue. IIM frequently affects other organs pointing to IIM as a systemic inflammatory disease. Cardiac involvement is associated with poor prognosis. The symptoms are often subclinical and therefore overlooked. QTC prolongation has been detected in patients with systemic sclerosis (SSc). Autoantibodies are important diagnostic tools to confirm IIM and are present in approx. $60 \%$ of IIM patients. Autoantibodies are increasingly being recognized as markers for specific organ involvement. A biomarker for cardiac involvement has yet to be elucidated.

Objectives: The aim is to generate new knowledge about cardiac involvement in IIM detected by electrocardiography (ECG) and to evaluate possible associations between autoantibodies and cardiac involvement detected on ECG in a cohort of IIM patients compared with ECG changes in a cohort of SSc patients.

Methods: In a Scandinavian cohort, 263 IIM patients (130 polymyositis patients, 77 dermatomyositis patients, and 56 inclusion body myositis patients) and 102 SSc patients were investigated by ECG and basic cardiovascular and disease specific assessments according to international guidelines. IIM patients were tested for myositis specific autoantibodies (MSAs; anti-Jo-1, anti-PL-7, anti-PL-12, anti-OJ, anti-EJ, anti-SRP, anti-Mi2, anti-MDA5, anti-TIF1 $\gamma$, anti-NPX2, anti-SAE1, anti-HMGCR) and myositis associated autoantibodies (MAAs; anti-PM/Scl75, anti-PM/Scl100, antiRo52, anti-Ku, anti-cN1A)

Results: Twenty two IIM patients (8.49\%) had abnormal QRS duration versus one SSc patient $(1.06 \%)(P=0.012)$. SSc patients had significantly longer QTC duration than IIM patients (QTc $=432.7 \mathrm{~ms} \pm 22.2$ and $426.4 \mathrm{~ms} \pm 23.6$, respectively) $(P=0.03)$.

Multivariate regression analysis revealed that increased $\mathrm{C}$-reactive protein $(\mathrm{CRP})(\mathrm{P}=0.008)$, gender $(\mathrm{P}=0.002)$, and hypertension $(P=0.007)$ were associated with QTC duration in IIM patients. Likewise, pulmonary arterial hypertension was associated with QTC duration $(P<0.001)$ in SSc patients.

In analysis of pooled data for IIM patients and SSc patients, factors associated with QTc duration were increased CRP $(P=0.005)$, gender $(P=0.001)$, and hypertension $(P=0.01)$.

Conclusion: Both IIM patients and SSc patients had ECG changes though no particular pattern was shown. The findings support our hypothesis on cardiac involvement in IIM patients. No significant association was found between presences of either myositis specific or myositis associated autoantibodies and ECG changes. This could be due to the relatively low number of each autoantibody. There is a need to conduct larger prospective studies to identify a possible autoantibody for cardiac involvement in IIM.

Acknowledgement: The authors would like to thank all participating patients and the study personnel at the including centres.

Disclosure of Interests: Sine Søndergaard Korsholm: None declared, Maryam Dastmalchi: None declared, John Bonde Knudsen: None declared, Axel Cosmus Diederichsen: None declared, Ingrid E. Lundberg Grant/ research support from: Dr. Lundberg has received honoraria from Bristol Myers Squibb and Medlmmune and is currently receiving a research grant from Bristol Myers Squibb and from Astra Zeneca., Consultant for: She is a scientific advisor for Bristol Myers Squibb, and aTyr, Daniel C Andersson: None declared, Nanna Witting: None declared, Søren Jacobsen: None declared, Tina Friis Grant/research support from: Anti cN-1A ELISA kits and EUROLINE Autoimmune Inflammatory Myopathies 16 AG kits have been provided for a project free of charge from Euroimmun. Markus E. Krogager: None declared, Louise Pyndt Diederichsen: None declared

DOI: 10.1136/annrheumdis-2019-eular.2394

\section{AB0653 SERUM CARDIAC BIOMARKERS BUT NOT SUBCLINICAL CARDIOVASCULAR MAGNETIC RESONANCE ABNORMALITIES IN SYSTEMIC SCLEROSIS ASSOCIATE WITH THE DEVELOPMENT OF CARDIOVASCULAR EVENTS}

Raluca-Bianca Dumitru ${ }^{1}$, Lesley Anne Bissell ${ }^{1}$, Bara Erhayiem ${ }^{2}$, Graham Fent $^{2}$, Ananth Kidambi $^{2}$, Peter Swoboda ${ }^{2}$, Helena Donica ${ }^{3}$, Agata Burska ${ }^{1}$, John Biglands ${ }^{4}$, John Greenwood ${ }^{2}$, Francesco Del Galdo ${ }^{1}$, Sven Plein ${ }^{2}$ Maya Buch'. ' ${ }^{1}$ Leeds Institute of Rheumatic and Musculoskeletal Medicine, Leeds, United Kingdom; ${ }^{2}$ Leeds Institute of Cardiovascular and Metabolic Medicine, Leeds, United Kingdom; ${ }^{3}$ Department of Biochemical Diagnostics, Lublin, Poland; ${ }^{4}$ National Institute for Health Research, Leeds Biomedical Research Centre, Leeds, United Kingdom

Background: Primary cardiac disease in systemic sclerosis (SSc-PCD) is associated with a poor prognosis when clinically evident. Subclinical SScPCD is described in up to $2 / 3$ of SSc patients when sensitive methods are employed. The prognostic implication of these findings is not clear. Objectives: To describe the prevalence of cardiovascular magnetic resonance (CMR) abnormalities, their association with clinical phenotype and cardiac biomarkers, and whether cardiac biomarkers and CMR predict the development of cardiovascular (CV) events.

Methods: Patients fulfilling the ACR/EULAR criteria for SSc, with no CV disease (CVD), diabetes and no more than $1 \mathrm{CV}$ risk factor had 3Tesla CMR, including late gadolinium enhancement (LGE), myocardial perfusion, T1 mapping for T1 native and extracellular volume (ECV) quantification, and cardiac biomarkers measured. CV events were defined as episode of myocarditis, heart failure, rhythm disturbances and/or any echocardio graphic abnormalities including systolic dysfunction, diastolic dysfunction > grade 1 or regional wall motion abnormalities. 47 healthy volunteers (HV) with comparable age and gender served as controls.

Results: 83 SSc patients were recruited, median (IQR) age of 54 (49, 54 ), $84 \%$ females and $33 \%$ dcSSc, $40 \%$ had ILD, $24 \%$ a history of digital ulceration (HxDU) and $29 \%$ were Scl70+.

CMR showed higher ECV\% and T1 native, markers of diffuse fibrosis in SSc compared to HV [mean (SD) 30 (4) vs 25 (3), $\mathrm{p}=0.000 ; 1241$ (76) vs 1206 (55), $p=0.003$ ]. Lower myocardial perfusion reserve (MPR) was also noted in SSc patients [mean (SD) $2(0.8)$ vs 3 (1), $\mathrm{p}=0.000$ ]. None of the HV had LGE-fibrosis, whilst $16(20 \%)$ of the patients had a nonischemic LGE pattern. Left ventricular (LV) volume and function were similar between HV and SSc patients.

Presence of LGE associated with hsTnl and lower forced vital capacity (rho=0.283, $\mathrm{p}=0.032$; $\mathrm{rho}=-0.217, \mathrm{p}=0.5$ ). Multivariate analysis revealed an association of $T 1$ native with mRSS and CRP $(B=0.5, p=0.02 ; B=0.218$, $\mathrm{p}=0.049)$. ECV\% associated with NTproBNP and HxDU $(B=0.032$ $p=0.034 ; \quad B=0.310, p=0.005)$. $73 / 83$ had $\geq 1$ year available clinical and echocardiography follow up data with a mean (SD) duration of 24(20) months. $12 / 73$ had a CV event judged to be SSc-PCD. LV mass associated with $C V$ events $(p=0.043)$ (Table 1$)$, whilst univariate regression analysis showed that both hsTnl (OR=1.014, $\mathrm{p}=0.014)$ and NTproBNP $(\mathrm{OR}=1.005, p=0.026)$ but none of the CMR variables associated with the development of $\mathrm{CV}$ events.

Conclusion: Asymptomatic SSc patients with no overt CVD demonstrate both focal and diffuse fibrosis, which are associated with cardiac bio markers and markers of SSc disease activity. Whilst hsTnl and NTproBNP associated with the development of CV events, none of the measures of fibrosis did although a higher LV mass was noted in this 